\title{
Onconeuronal and Antineuronal Antibodies in Patients with Neoplastic and Non-Neoplastic Pulmonary Pathologies and Suspected for Paraneoplastic Neurological Syndrome
}

\author{
S. Michalak ${ }^{1,2}$, S. Cofta ${ }^{3}$, A. Piatek ${ }^{3}$, J. Rybacka ${ }^{3}$, E. Wysocka ${ }^{4}$, W. Kozubski ${ }^{5}$ \\ ${ }^{1}$ Department of Neurochemistry and Neuropathology, Poznan University of Medical Sciences, Poznan, Poland; ${ }^{2}$ Neuroimmunological Unit, \\ Polish Academy of Sciences, Poznan, Poland; ${ }^{3}$ Department of Respiratory Medicine, ${ }^{4}$ Department of Clinical Biochemistry and Laboratory \\ Medicine, and ${ }^{5}$ Department of Neurology, Poznan University of Medical Sciences, Poznan, Poland
}

\begin{abstract}
Objective: Onconeuronal antibodies are important diagnostic tool in patients with suspicion of paraneoplastic neurological syndromes (PNS). However, their role in PNS pathophysiology and specificity for particular neurological manifestation remains unclear. The aim of this study was to evaluate onconeuronal and antineuronal antibodies in patients with pulmonary pathologies and suspected for PNS.

Material and methods: Twenty one patients with pulmonary pathologies were selected from the database of 525 consecutive patients with suspicion of PNS. Patients' sera were screened for the presence of onconeuronal and antineuronal antibodies by means of indirect immunofluorescence; the presence was confirmed by Western blotting. Clinical data were obtained from medical records, hospital data base, and questionnaire-based direct telephone contact with patients.
\end{abstract}

Results: Among 21 patients, aged $54 \pm 11$, with pulmonary pathologies, the most frequent neurological manifestations were neuropathies. Typical PNS included paraneoplastic cerebellar degeneration (PCD) and limbic encephalitis (LE). We found cases with multiple onconeuronal antibodies (anti-Ri and anti-Yo) and coexisting PNS (PCD/LE). Well-defined onconeuronal antibodies were identified in $23.8 \%$ of patients. Among antineuronal antibodies, the most frequent were antiMAG (23.8\%). ROC curves analysis revealed high sensitivity of onconeuronal and antineuronal antibodies for typical PNS and lower for pulmonary malignancies.

Conclusions: Tests for antibodies are highly sensitive for the diagnosis of typical paraneoplastic neurological syndromes. Anti-myelin and anti-MAG antibodies are associated with non-neoplastic pulmonary diseases. Patients with well-defined onconeuronal antibodies require careful screening and follow-up, because the PNS diagnosis indicates a high probability of an underlying malignancy.

Key words: onconeuronal antibodies, antineuronal antibodies, lung cancer, paraneoplastic neurological syndromes, bronchial asthma, chronic obstructive pulmonary disease

\section{INTRODUCTION}

Paraneoplastic neurological syndromes (PNS) are defined as remote effects of systemic malignancy and autoimmune reactions are currently an accepted hypothesis of the underlying pathophysiology. Since 2004, when Graus et al [1] published the PNS diagnostic criteria, the following notions were introduced in clinical and laboratory practice: typical paraneoplastic syndromes and well-defined antibodies. According to Graus et al criteria [1], a definite PNS diagnosis can be made in subjects with:

1. typical PNS and/or onconeuronal antibodies with or without identification of systemic malignancy; and

2. non-typical PNS and onconeuronal antibodies with or without identification of systemic malignancy.

Thus, the presence of onconeuronal antibodies remains crucial for the diagnosis of paraneoplastic neurological syndromes. Typical syndromes include: limbic encephalitis, paraneoplastic cerebellar degeneration, Lambert-Eaton myasthenic syndrome, subacute sensory neuropathy, opsoclonus/myoclonus and dermatomyositis [1]. Well-defined onconeuronal antibodies are anti-Hu, anti-Ri, anti-Yo, anti-Ma/Ta, anti-Cv2, and anti-amphiphysin [1] are identified by means of Western blotting with the use of recombinant proteins. Antibodies causing positive reaction on indirect immunofluorescence, without confirmation by Western blotting, do not satisfy the definition of well-defined.

Routine indirect immunofluorescence tests enable identification of antineuronal antibodies like antimyelin, anti-myelin-associated glycoprotein (antiMAG), and anti-glutamic acid decarboxylase (antiGAD). Paraneoplastic neurological syndromes have been reported in $4-5 \%$ of lung cancer patients [2]. Seute et al [3] found seven Lambert-Eaton myasthenic syndrome cases $(1.6 \%)$, polyneuropathy in two cases $(<1 \%)$, subacute cerebellar degeneration in one case $(<1 \%)$, and limbic encephalitis in three cases $(<1 \%)$ among 432 small cell lung cancer patients. Recent therapeutic recommendations do not exclude patients with lung cancer and PNS from potentially effective treat- 
ment on the basis of the symptoms alone [4]. The pathomechanism believed to be responsible for the development of paraneoplastic neurological syndromes is immune-mediated. A broad spectrum of antibodies has been described in cancer patients with neurological symptoms. For the first time, Wilkinson and Zeromski [5] in 1965 identified antineuronal antibodies in a patient with sensory neuropathy in the course of lung cancer. As mentioned above, the evaluation of onconeuronal antibodies is an important step in PNS diagnosis. Up to $20 \%$ of patients with small-cell lung cancer have anti-Hu antibodies [6]. Lung cancer is associated with other onconeuronal antibodies reported: anti-CV2 [7], anti-Ri [8], and anti-amphiphysin [9]. On the other hand, anti-GAD antibodies were reported in stiff-man syndrome patient, who developed West Nile Fever and had also history of bronchial asthma [10]. The coexistence of different autoimmune disorders and overlapping of co-morbidities needs to be taken into consideration in differential diagnostics.

The aim of the present study was to evaluate onconeuronal and antineuronal antibodies in patients with neoplastic and non-neoplastic pulmonary pathologies and suspected for paraneoplastic neurological syndromes.

\section{Material AND Methods}

A local Bioethics Committee at the University of Medical Sciences in Poznan, Poland approved this clinical study.

From the database of 525 consecutive patients with suspicion of neurological paraneoplastic syndromes we have selected 21 patients with pulmonary diseases. At the onset of neurological deficit, the patients' sera were tested for the presence of onconeuronal (anti$\mathrm{Hu}$, anti-Ri, anti-Yo, anti-Ma/Ta, anti-Cv2, and antiamphiphysin) and antineuronal antibodies (antimyelin, anti-MAG, and anti-GAD). The screening was performed by means of indirect immunofluorescence and the presence of onconeuronal antibodies (anti$\mathrm{Hu}$, anti-Ri, anti-Yo, anti-Ma/Ta, anti-Cv2, and antiamphiphysin) was confirmed by Western blotting with recombinants (EUROIMMUN, Luebeck, Germany). Antineuronal antibodies (anti-myelin, anti-MAG, and anti-GAD) were tested by means of indirect immunofluorescence only (EUROIMMUN, Luebeck, Germany), if there was no confirmation test available. Thus, onconeuronal antibodies tested in our study satisfied the definition of well-defined antibodies. A neurological paraneoplastic syndrome was diagnosed on the basis of Graus et al's criteria [1].

Clinical data analyzed in the patients included in the study involved the diagnosis of pulmonary pathology (neoplastic or non-neoplastic), paraneoplastic neurological syndrome (typical or non-typical), age, gender, and 2 to 5-year follow-up. Data for follow-ups were obtained from medical records, hospital data base, and direct phone contact with patients. The phone data were collected on the basis of a questionnaire prepared by the authors (J.R. \& A.P.).

\section{STATISTICAL ANALYSIS}

The exact Fisher test and a t-test were used for statistical analyses. ROC (receiver operating characteristic) curves were construed to evaluate sensitivity and specificity of onconeuronal and antineuronal tests. $\mathrm{P}<0.05$ was accepted as indicative of significant differences. All statistical analyses were performed with the use of MedCalc Statistical Software version 10.3.1.0.

\section{RESULTS}

Twenty one patients aged $54 \pm 11$ with pulmonary diseases were selected from 525 patients with suspicion of a neurological paraneoplastic syndrome. There was no significant difference in the age of males $(n=6)(56$ $\pm 14)$ and females $(n=15)(53 \pm 10)$. The gender ratio of females to males was 2.5. The most frequent neurological manifestations were neuropathies $(n=8 ; 38 \%)$ (Table 1). Typical PNS included paraneoplastic cerebellar degeneration (PCD) $(n=1 ; 4.8 \%)$ and limbic encephalitis $(\mathrm{LE})(\mathrm{n}=2 ; 9.5 \%)$. In one case, LE coexisted with PCD as it was observed both clinically and on neuroimaging (Fig. 1 - Panels A, B, and C). It is note-
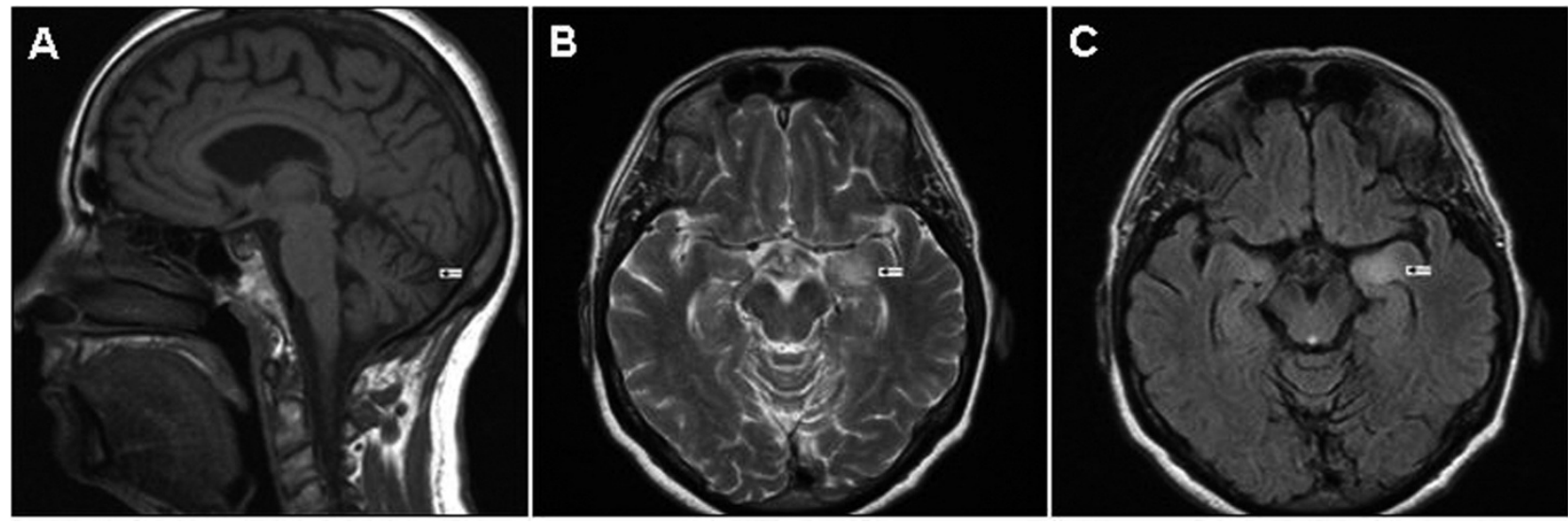

Fig. 1. Panel A - MRI of a patient with LE/ PCD and anti-amphiphysin antibodies, T1-weighted image reveals cerebellar atrophy $(\rightarrow)$; Panel B - MRI of a patient with LE/PCD and anti-amphiphysin antibodies, T2-weighted image shows hyperintensity $(\rightarrow)$ typical for limbic encephalitis; and Panel C - MRI of the patient with LE / PCD and anti-amphiphysin antibodies, FLAIR image shows hyperintensity $(\rightarrow)$ typical for limbic encephalitis. 
Table 1. Clinical data of 21 patients with pulmonary pathology selected from 525 subjects with suspicion of paraneoplastic neurological syndromes.

\begin{tabular}{|c|c|c|c|c|c|}
\hline Patient & Age & Gender & Antibody & NPS & Pulmonary disease \\
\hline J.F. & 56 & Male & Negative & Motor neuron disease & Small-cell lung cancer \\
\hline J.W. & 52 & Male & Anti-Ri, anti-Yo & Neuropathy & Chronic obstructive pulmonary disease \\
\hline B.K. & 54 & Female & Negative & Neuropathy & Non-small-cell lung cancer, brain metastases \\
\hline K.K. & 55 & Female & Negative & Neuropathy & Non-small-cell lung cancer \\
\hline S.P. & 75 & Male & Anti-Hu & Limbic encephalitis & Small-cell lung cancer \\
\hline E.S. & 42 & Female & Negative & Pyramidal syndrome & Non-small-cell lung cancer \\
\hline A.S. & 41 & Male & Negative & Pyramidal syndrome & Pulmonary sarcoidosis \\
\hline S.K. & 31 & Female & Anti-myelin & Cerebellar syndrome & Bronchial asthma \\
\hline W.J. & 57 & Female & Anti-MAG & Neuropathy & Bronchial asthma \\
\hline A.S. & 51 & Female & Negative & Polymyositis & Non-small-cell lung cancer, ovarian cancer \\
\hline K.G. & 58 & Female & Anti-MAG & Neuropathy & Small-cell lung cancer \\
\hline H.R. & 47 & Female & Anti-Hu & Pyramidal syndrome & Small-cell lung cancer \\
\hline K.L. & 60 & Female & Anti-Ri & Neuropathy & Breast cancer, pulmonary metastases \\
\hline I.N. & 53 & Female & Negative & Brachial plexopathy & Pulmonary metastases \\
\hline T.Z. & 70 & Female & Negative & Myopathy & Chronic obstructive pulmonary disease \\
\hline A.S. & 41 & Male & Negative & Pyramidal syndrome & Pulmonary sarcoidosis \\
\hline W.J. & 57 & Female & Anti-MAG & Neuropathy & Bronchial asthma \\
\hline I.B. & 38 & Female & Anti-MAG & Myasthenia gravis & Bronchial asthma \\
\hline F.C. & 68 & Male & Anti-MAG & Neuropathy & Small-cell lung cancer \\
\hline A.K. & 52 & Female & Negative & Neuropathy & Small-cell lung cancer, brain metastases \\
\hline A.B. & 69 & Female & Anti-amphiphysin & $\begin{array}{l}\text { Limbic encephalitis, } \\
\text { paraneoplastic } \\
\text { cerebellar } \\
\text { degeneration. }\end{array}$ & Non-small-cell lung cancer \\
\hline
\end{tabular}

Table 2. Number of patients with or without pulmonary malignancy and the presence of onconeuronal, antineuronal, and welldefined onconeuronal antibodies.

\begin{tabular}{lcc}
\hline & Primary pulmonary malignancy & No primary pulmonary malignancy identified \\
\hline $\begin{array}{l}\text { Onconeuronal and antineuronal } \\
\text { antibodies }(\mathrm{n}=11)\end{array}$ & 5 & 6 \\
$\begin{array}{l}\text { No onconeuronal and antineuronal } \\
\text { antibodies present }(\mathrm{n}=10)\end{array}$ & $(45 \%)$ & $(54 \%)$ \\
$\begin{array}{l}\text { Well-defined onconeuronal } \\
\text { antibodies ( }=5)\end{array}$ & $(60 \%)$ & 4 \\
$\begin{array}{l}\text { No well-defined onconeuronal } \\
\text { antibodies present }(\mathrm{n}=16)\end{array}$ & $(60 \%)$ & $(40 \%)$ \\
& 8 & 8 \\
\end{tabular}

worthy that in this case we found the presence of antiamphiphysin antibodies and non small-cell lung cancer was diagnosed.

In one case with neuropathy, we observed a co-existance of two onconeuronal antibodies (anti-Ri and anti-Yo). Well-defined onconeuronal antibodies were identified in 5 patients $(23.8 \%$ ) (Table 1$)$. Among antineuronal antibodies, the most frequent were antiMAG ( $\mathrm{n}=5 ; 23.8 \%)$. Interestingly, anti-myelin and anti-MAG were associated with bronchial asthma, the most frequent non-neoplastic respiratory tract disease in our patients. There were no significant differences in the number of the patients with or without onconeuronal and antineuronal antibodies and pulmonary malignancy, nor were there any in the patients with or without well-defined onconeuronal antibodies and pulmonary malignancy (Table 2).

Comparison of ROC curves for all onconeuronal and antineuronal antibodies with well-defined onconeuronal antibodies did not show significant differences (Fig. 2). Nor were there any significant differences between ROC curves for onconeuronal and an- 


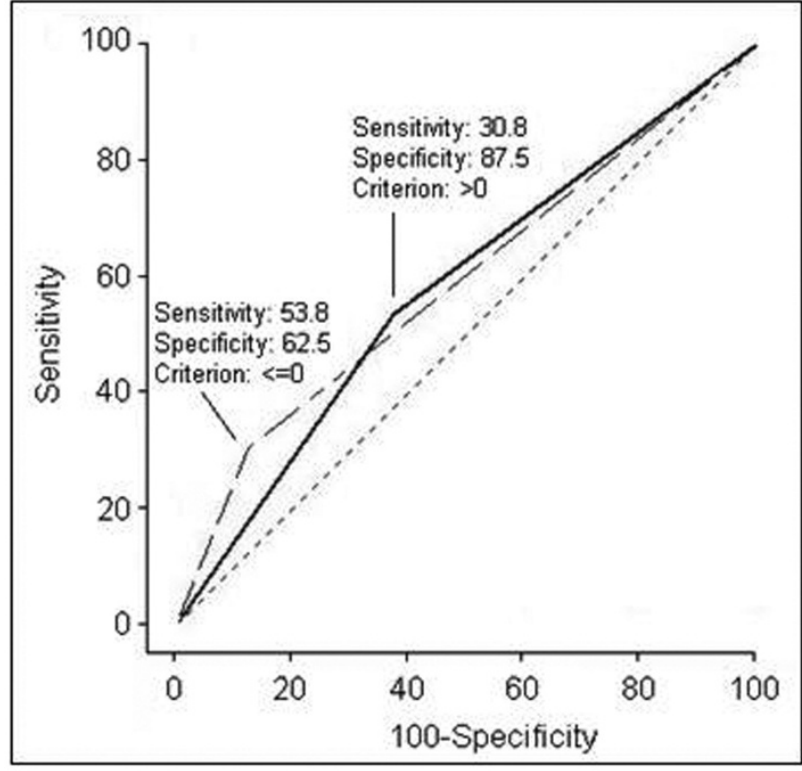

Fig. 2. Comparison of ROC curves for onconeuronal and antineuronal antibodies (solid line) and well-defined onconeuronal antibodies (dashed line) in patients with and without pulmonary malignancy.

tineuronal antibodies and well-defined onconeuronal antibodies in the patients with or without typical PNS (Fig. 3). However, the sensitivity of the diagnosis of a typical PNS reached 100\% for onconeuronal and antineuronal antibodies.

\section{DISCUSSION}

Clinical significance of onconeuronal antibodies is limited to the use of indirect immunofluorescence and Western blotting in the diagnostic procedures of paraneoplastic neurological syndromes. Despite advanced immunological studies that have revealed antibodies acting against structures of the nervous system, the pathomechanism of neurological paraneoplastic syndromes remains incompletely recognized. Some reports [11] address the onconeuronal antibodies as coexisting with and predicting cancer, rather than inducing a neurological syndrome. Seronegative patients manifesting typical clinical paraneoplastic syndromes fulfill Graus's criteria for a definite diagnosis, when systemic malignancy is identified. Furthermore, immunosuppressive treatment [12] and intravenous immunoglobulins [13] are clinically ineffective, indicating an involvement of a different, probably metabolic pathomechanism. The latter possibility is suggested by the clinical observation of a coexisting myasthenic Lambert-Eaton syndrome and subacute paraneoplastic degeneration. Graus et al [14] noted an improvement of myasthenic symptoms as a result of an immunomodulating treatment, while the symptoms of coexisting subacute paraneoplastic cerebellar degeneration persisted. Experimental studies suggest metabolic effects of systemic malignancy on the cerebellum [15], spinal cord [16], and peripheral nerves [17].

In the present study, we analyzed onconeuronal and

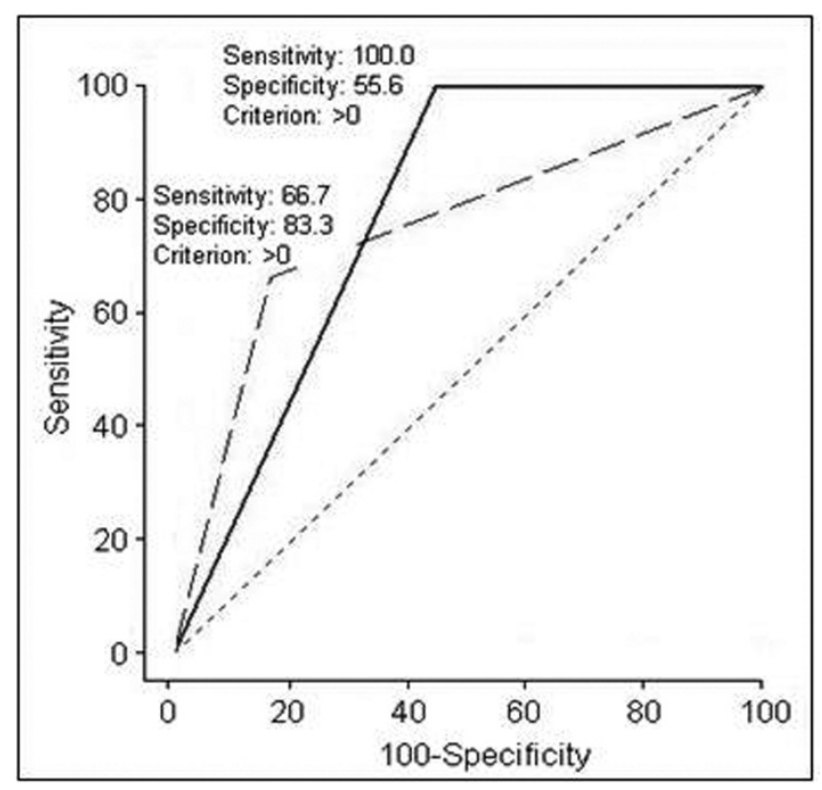

Fig. 3. Comparison of ROC curves for onconeuronal and antineuronal antibodies (solid line) with well-defined onconeuronal antibodies (dashed line) in patients with and without typical paraneoplastic neurological syndrome.

antineuronal antibodies in patients with pulmonary pathologies selected from subjects with suspicion of paraneoplastic neurological syndromes. Sensitivity of onconeuronal and antineuronal antibodies evaluation in patients with and without pulmonary malignancy was higher $(53.8 \%)$ than that for well-defined onconeuronal antibodies (30.8\%), although the specificity of well-defined onconeural antibodies was better $(87.5 \%)$. Comparison of ROC curves for both tests did not show any significant difference. The best sensitivity of the diagnosis of typical paraneoplastic syndrome $(100 \%)$ for onconeuronal and antineuronal antibodies is noteworthy and indicates usefulness of those tests for PNS, rather than for pulmonary malignancy. This is in opposition to Pittock et al's [11] suggestions mentioned above. However, the population examined in that study consisted of all patients with PNS suspicion, while in our present study we evaluated subjects with pulmonary disease only. In our study we found multiple antibodies in 1 case $(4.8 \%)$ only, while in studies involving patients with a spectrum of malignancies $31 \%$ of cases had more than one antibody [11]. Those authors [11] stated that the presence of multiple onconeuronal antibodies suggests autoimmune reaction directed against antigens localized at different levels of the nervous system, which leads to multifocal signs. According to Graus et al's diagnostic criteria [1], onconeuronal antibodies are significant for definite diagnosis of PNS as such, not for a specific syndrome. That was also the case in the present study showing high sensitivity of antibodies for the diagnosis of typical PNS, rather than for malignancy. Moreover, onconeuronal antibodies are not recognized as markers of malignancy in clinical practice.

In our group of patients, we also observed one case $(4.8 \%)$ of multiple typical paraneoplastic neurological 
syndromes, a paraneoplastic cerebellar degeneration and limbic encephalitis. Several case studies show coexistence of PNS: paraneoplastic cerebellar degeneration with Lambert-Eaton myasthenic syndrome [14], or limbic encephalitis with neuropathy or with paraneoplastic cerebellar degeneration [18]. Multifocal PNS may suggest multiple onconeuronal antibodies; however, in the present study, the patient with PCD and LE had anti-amphiphysin antibodies only.

We also found non-neoplastic diseases, e.g., bronchial asthma associated with the presence of antimyelin and ant-myelin-associated glycoprotein antibodies. Overlapping and/or coexistence of autoimmune reactions are well known from clinical practice. No well-defined onconeuronal antibodies were found in non-neoplastic pulmonary diseases.

Autoimmunity has been reported as a source of factors modulating angiogenesis, and proangiogenic effects of sera from patients with systemic diseases depend on the intensity of radiological signs of a pulmonary pathology [19]. On the other hand, the intensity of proangiogenic stimulation does not correlate with dyspnea or lung function tests [19]. However, a suggestion for an association of autoimmunity, pulmonary pathology, and modulation of angiogenesis has been made [19]. Proangiogenic factors, such as vascular endothelial growth factor (VEGF), enhance vascular permeability and increase blood-brain barrier leakage and these factors' expression is increased in autoimmune central nervous system diseases [20]. Thus, autoimmunity directed against the nervous system associated with pulmonary pathology could, via stimulation of angiogenesis, facilitate paraneoplastic reaction.

In summary, both onconeuronal and antineuronal antibodies are associated with pulmonary diseases. The evaluation of onconeuronal and antineuronal antibodies is highly sensitive for the diagnosis of typical paraneoplastic neurological syndromes. Lower specificity of onconeuronal and antineuronal antibodies tests indicate complex autoimmune reactions observed in paraneoplastic neurological syndromes and the possibility of nonimmune-mediated pathomechanisms. Anti-myelin and anti-MAG antibodies are associated with non-neoplastic pulmonary diseases. Careful screening and follow-up are needed for patients with well-defined onconeuronal antibodies, because the diagnosis of paraneoplastic neurological syndromes indicates a high probability of an underlying malignancy.

Conflicts of interest: The authors declared no conflicts of interest in relation to this article.

\section{REFERENCES}

[1] Graus F, Delattre JY, Antoine JC, Dalmau J, Giometto B, Grisold W, Honnorat J, Sillevis Smitt P, Vedeler Ch, Verschuuren JJ, Vincent A, Voltz R, for the Paraneoplastic Neurological Syndrome Euronetwork. Recommended diagnostic criteria for paraneoplastic neurological syndromes. J Neurol Neurosurg Psych 2004; 75: 1135-40.

[2] Swash M, Schwartz MS. Paraneoplastic syndromes. In: Johnson RT (ed). Current Therapy in Neurologic Diseases. BC Decker, Philadelphia 1990, 236-43.
[3] Seute T, Leffers P, ten Velde GPM, Twijnstra A. Neurologic disorders in 432 consecutive patients with small cell lung carcinoma. Cancer 2004; 100: 801-6.

[4] Spiro SG, Gould MK, Colice GL. Initial evaluation of the patient with lung cancer: symptoms, signs, laboratory tests, and paraneoplastic syndromes. Chest 2007; 132: 149S-60S

[5] Wilkinson PC, Zeromski J. Immunofluorescent detection of antibodies against neurones in sensory carcinomatous neuropathy. Brain 1965; 88: 529-83.

[6] Anderson NE, Rosenblum MK, Graus F, Wiley RG, Posner JB. Autoantibodies in paraneoplastic syndromes associated with small-cell lung cancer. Neurology 1988; 38: 1391-98.

[7] Yu Z, Kryzer TJ, Griesmann GE, Kim K, Benarroch EE, Lennon VA. CRMP-5 neuronal autoantibody: marker of lung cancer and thymoma-related autoimmunity. Ann Neurol 2001; 49: 146-54.

[8] Moll JW, Antoine JC, Brashear HR, Delattre J, Drlicek M, Dropcho EJ, Giometto B, Graus F, Greenlee J, Honnorat J. Guidelines on the detection of paraneoplastic antineuronal-specific antibodies: Report from the Workshop to the Fourth Meeting of the International Society of Neuro-Immunology on paraneoplastic neurological disease. Neurology 1995; 45(10):1937-41.

[9] Saiz A, Dalmau J, Husta Butler M, Chen Q, Delattre JY, De Camilli P, Graus F. Anti-amphiphysin I antibodies in patients with paraneoplastic neurological disorders associated with small cell lung carcinoma. J Neurol Neurosurg Psych 1999; 66: 214-17.

[10] Hassin-Baer S, Kirson ED, Shulman L, Buchman AS, Bin H, Hindiyeh M, Markevich L, Mendelson E. Stiff-person syndrome following West Nile fever. Arch Neurol 2004; 61: 938-41.

[11] Pittock SJ, Kryzer TJ, Lennon VA. Paraneoplastic antibodies coexist and predict cancer, not neurological syndrome. Ann Neurol 2004; 56: 715-19.

[12] Keime-Guilbert F, Grauss F, Broët P, Rene R, Molinuevo JL, Ascaso C, Delattre JY. Clinical outcome of patients with anti-Hu-associated encephalomyelitis after treatment of the tumor. Neurology 1999; 53: 1719-26.

[13] Keime-Guilbert F, Graus F, Fleury A, René R, Honnorat J, Broet P, Delattre J-Y. Treatment of paraneoplastic neurological syndromes with antineuronal antibodies with a combination of immunoglobulins, cyclophosphamide, and methylprednisolone. J Neurol Neurosurg Psych 2000; 68: 479-82.

[14] Grauss F, Lang B, Pozo-Rosich P, Saiz A, Casamitjana R, Vincent A. P/Q type calcium channel antibodies in paraneoplastic cerebellar degeneration with lung cancer. Neurology 2002; 59: 764-66.

[15] Michalak S, Wender M, Michalowska-Wender G. Cachexia-induced cerebellar degeneration: involvement of serum TNF and MCP-1 in the course of experimental neoplastic disease. Acta Neurobiol Exp 2006; 66(2): 113 22.

[16] Michalak S, Ambrosius W, Osztynowicz K, Kozubski W. ATP-ases activities, lipids and heat shock protein $70 \mathrm{kDa}$ in spinal cord in rats with experimental neoplastic disease. Res J Med Sci 2007; 1(4): 199-208.

[17] Michalak S, Adamczewska-Goncerzewicz Z, Szczech J. ATPases and lipid peroxidation in the rat sciatic nerve in the course of experimental neoplastic disease. Exp Mol Pathol 2006; 81(1): 92-99.

[18] Gultekin SH, Rosenfeld MR, Voltz R, Eichen J, Posner JB, Dalmau J. Paraneoplastic limbic encephalitis: neurological symptoms, immunological findings and tumor association in 50 patients. Brain 2000; 123: 1481-94.

[19] Zielonka TM, Demkow U, Zycinska K, Filewska M, Korzeniewska M, Radzikowska E, Bialas-Chromiec B, Kus J, Wardyn KA, Skopinska-Rozewska E. Angiogenic activity 
of sera from patients with systemic autoimmune diseases in relation to clinical, radiological, and functional pulmonary status. J Physiol Pharmacol 2008; 59 Suppl 6: 791-800. [20] Argaw AT, Zhang Y, Snyder BJ, Zhao ML, Kopp N, Lee SC, Raine CS, Brosnan CF, John GR. IL-1 regulates blood-brain barrier permeability via reactivation of the hypoxia-angiogenesis program. J Immunol 2006; 177: 5574-84.
Corresponding author:

Slawomir Michalak

Przybyszewskiego St. 49

60-355 Poznan

Poland

Phone: +48618691443

Fax: $\quad+48618691444$

E-mail: swami622@gmail.com 